\title{
Evaluation the Role of Micro-RNAs During Stages of Breast Cancer: A Review
}

\author{
Haider Y. Lafta ${ }^{1}$, Risala H. Allami², Sahar M. Hussein ${ }^{3}$ \\ ${ }^{1}$ Assistant Lecturer, Ministry of Science and Technology, Central Labs Directorate, \\ ${ }^{2}$ Assistant Prof., ${ }^{3}$ Lecturer, Al-Nahrian University-College of Biotechnology, Baghdad, Iraq
}

\begin{abstract}
Breast cancer is one of the most deadly cancers threatening life and is the most prevalent among women worldwide and in general is the second most common type of cancer. The aim of this study was to identify the expression of microRNAs and biomarkers for early detection of breast cancer. MicroRNAs have become a hot subject in cancer research today because of their important role not only in cancer progression, progression, But also suppress genes related to cancer. With advanced techniques, these microRNAs can be easily detected from biopsy samples and blood for early diagnosis, diagnosis and treatment. Due to the increase in the demand for research in the exploration of the microRNAs profile in relation to different subtypes of breast cancer, the role of microRNA microRNA as an oncomir or oncosupressor, and discovery of microRNAs and miRNAs potentials for the treatment of breast cancer. MiR is involved in a variety of natural physiological processes, specifically identifying and identifying cell type. It has become clear that they are also major factors in cancer. Here we discuss what is known about miR biology in natural breast, and the emerging roles in breast cancer.
\end{abstract}

Keywords: Breast Cancer, miRNAs, Target prediction, Gene expression profile.

\section{Introduction}

Breast cancer remains as one of the deadliest disease worldwide $^{(1)}$. High throughput studies highlighted the role of different sets of miRNAs in the control of gene expression during and after initiation stages of breast cancer as well as metastasizes stage ${ }^{(2)}$. However, a comprehensive overview and classification of the most important miRNAs at different stages of this type of cancer still remains to be elucidated. Here, using different tools and web resources we have listed the most important miRNAs that appear to be involved during different stages of breast cancer ${ }^{(1,4)}$. Besides, we have predicted the most probable target genes for the proposed miRNAs and assessed the gene expression status of those targets in this type of cancer. Then we have profiled the gene

\section{Corresponding Author:}

\section{Risala H. Allami}

Assistant Prof., Al-Nahrian University-College of

Biotechnology, Baghdad, Iraq

e-mail: ririallami@yahoo.com expression patterns for these miRNAs and their target to gain an overview of the biological significance of these miRNAs in onset and progression of breast cancer. The results indicate usefulness of our approach in identifying novel miRNAs candidates related to breast cancer for possible lab work experiments.

Breast cancer is one of the most common malignancies in women, and represents the second leading to death after heart failure worldwide ${ }^{(5)}$. In fact, Iraq breast cancer positions the first amongst the communal malignancies amongst all the people and accounts for roughly one- third of the registered female cancer ${ }^{(6)}$, which highlights the importance of early detection. The finding of breast cancer in women represents the major problems for early detection, accurate staging and checking of breast cancer ${ }^{(7)}$. Thus, there is still need to develop a cost-effective and accurate screening method for this cancer and discover new biomarkers to improve diagnosis, prognosis and prediction (8),(9). As mentioned in American Cancer Society ${ }^{(10)}$ the objective of screening for early breast cancer discovery is to find the cancer before it starts to 
cause symptoms. Screening used to find a disease, such as cancer, in people who do not have any symptoms. Early discovery means consuming an approach that lets breast cancer get diagnosed earlier than otherwise might have happened.

Breast cancer can be detected by watchful physical examination, mammography, ultrasound (U/S), and magnetic resonance imaging (MRI) and breast biopsy ${ }^{(11)}$. With recent technological improvements, gene expression profiling is used also to detect early breast cancer and predict their prognostic results ${ }^{(12)}$.

MicroRNAs, which are novel class of naturally occurring, evolutionarily conserved, small RNA ranged in size 19-23 nucleotides, non-coding RNA molecules and are important in gene regulation and expression either by inhibition of translation or degradation of $\operatorname{mRNA}^{(13,14)}$.

Breast cancer currently is the public female malignancy in practically all of Asia, Europe and North America ${ }^{(15)}$ recommended being the first reason of death from cancers in women in Europe. Each year more than 1.3 million women were identified with breast cancer worldwide and about 465,000 die from the disease ${ }^{(16)}$. Although the fact that breast cancer is highly curable if diagnosed and preserved applicably at an early stage (17) . The increasing incidence of breast cancer results in a critical need to identify and develop more special means of diagnosing and treating the disease.

Aim of the Study: The overarching aims of this study were to first investigate the factors impacting the potential of circulating miRNAs as biomarkers for breast cancer.

MicroRNA and Cancer: The first studies about miRNA expression in human cancer examined a recurring deletion at chromosome $13 \mathrm{q} 14$ to search for tumor suppressor genes involved in chronic lymphatic leukemia $(C L L)^{(18)}$. The result shows that this region at the chromosome was encoded two miRNAs; miR-15a and miR-16-1, and the scientists then confirmed that these two miRNAs have a role in CLL pathogenesis ${ }^{(19)}$ found that genes for miRNA were positioned at fragile sites in the genes that are frequently deleted or amplified in human cancer. The data of the study provide a catalogue of miRNA genes that may have roles in cancer and argue that the full complement of miRNA in the genome may be involved in cancer.
Any altered expressions in levels of miRNA have been correlated in different studies with cancer type, tumor stage and response to treatment. These studies confirm that miRNA represents a new class of biomarker as a diagnostic and prognostic and cancer therapy ${ }^{(20)}$, miRNA-196 and miRNA-10a were shown to be located in home box clusters, which is identified to be convoluted in carcinogenesis and it is attendant with the malignant of cancer cell ${ }^{(21)}$. Each miRNA is thought to target multiple genes this will affect cellular process and may induce carcinogenesis. Up and down regulation of miRNA expression were observed in tumor compared to normal tissues, this indicate that microRNA play as either oncomiRs or tumor suppressors ${ }^{(22)}$. Usually, encoding miRNAsgenes were positioned on chromosomal regions that are overexpressed or amplified in cancer and function as oncogenes, while those are deleted or less expressed in cancer act as tumors suppressor miRNAs ${ }^{(23)}$. Over expression of miR-21 were observed in all cancers, which support the evidence of the role of this miR in malignancies and its oncomiR activity. And there are numbers of tumor suppressor genes were targeted by this oncomiR like PTEN ${ }^{(24)}$.

MicroRNA and Breast Cancer: The first studies that determine the global miRNA expression profiles in breast cancer used 76 breast tumors and 34 normal $^{(25)}$. The result of the study showed that there are many miRNA which down-regulated in these breast tumors like; miR-10b, miR-125b and miR-145 while others were shown to be up-regulated like; miR-21and miR155. Another finding from this study, it can differentiate between normal and cancer breast tissues. One of the most studied microRNA is miR-21 which was found play role as an oncogene in different cancers and in breast cancer especially ${ }^{(26)}$. Knockdown of miR-21 in MCF7 breast cancer cell lines was shown to reduce the growth and reduce tumour growth in mouse xenograft ${ }^{(27)}$. While miR-21 overexpression was correlated with disease aggressiveness and tumour grade ${ }^{(28)}$. By comparing the differentiation levels of miRNA expression in tumour cells, it could be expected that their levels were affected by methylation and demethylation of miRNA promoter regions during the development of cancer. However, miRNA may mutate in breast cancer, which may affect the detection of its expression. So, the study of Wang and Wei ${ }^{(29)}$, declared that genomic instability, epigenetic changes and mutation of miRNA cause miRNA deregulated in breast cancer. The function of miRNA in cancer invasion and metastasis were found the role 
of two miRNAs, miR-373 and miR-520c, in promoting invasion and metastasis of MCF7 cell lines ${ }^{(30)}$. On the other hand, miRNA levels and expression were measured in serum of breast cancer patients and used to differentiate between healthy and cancer individual (31); and concluded from this study that serum microRNA could be used as a biomarker in early stage breast cancer. In a study of Schrader and his group ${ }^{(32)}$, they used microRNA microarray technology to investigate miRNA expression in whole blood of early-stage breast cancer patients. The result indicated that miRNA need a diagnostic and prognostic possible in those patients compared to normal.

The stability of microRNAs, tissue-specific expression profiles and the easy to be quantified, suggest that these molecules represent an ideal biomarker for cancer. Elevated level expression of miR-195 with let7a and miR-155 in the blood of breast cancer patients increase the specificity and sensitivity of the test ${ }^{(33)}$. The result at the end concluded the potential utility of microRNA as unique and noninvasiveness breast cancer markers. The research to identify more efficient miRNA biomarkers in blood is a nonstop process. The latest publications suggest that miR-92a in the circulation of serum breast cancer patient was elevated and correlates with tumour size and lymph node metastases (34). The study gave some clues for improving diagnosis, prognosis and therapeutic strategy for the future of cancer. Another novel microRNA, miR-155 was shown important in serum of breast cancer ${ }^{(35)}$. This study analyzed miR-155 in the serum of breast cancer patients and showed that it was up regulated and this elevation was correlated with clinical stage and p53 status.

Character of breast cancer miRNA: Though widespread study mechanisms of molecular complicated in breast cancer are completed over the periods, experiments quiet triumph in the early identification and management patients of breast cancer, such as random response and improvement resistance to adjuvant therapies. miRNAs, as mRNA regulators, might help as original investigative and prognostic applicants, and therapeutic targets potent. Since 2005, the deregulation of miRNA in breast cancer was first described ${ }^{(36)}$. Also there are numerous studies on various miRNAs expression and its function in breast cancer as summarized in Table 1.

Table (1): miRNAs purpose as tumor suppressors or oncogenes in breast cancer.

\begin{tabular}{|l|l|l|}
\hline miRNAs & Targets & Functional pathways \\
\hline Tumor suppressor miRNAs & \\
\hline miR-206 & ESR1 & ER signaling \\
\hline miR-17-5p & AlB1,CCND1,E2F1 & Proliferation \\
\hline miR-125a,b & HER2,HER3 & Anchorage-dependent growth \\
\hline miR-200c & BM11,ZEB1,ZEB2 & TGF-beta signaling \\
\hline let-7 & H-RAS, HMGA2, LIN28,PEBP1 & Proliferation,differetiation \\
\hline miR-34a & CCND1,CDK6,E2F3,MYC & DNA damage, proliferation \\
\hline miR-31 & $\begin{array}{l}\text { FZD3,ITGA5,M- } \\
\text { RIP,MMP16,RDX,RHOA }\end{array}$ & Metastasis \\
\hline miR-335 & SOX4,PTPRN2,MERTK,TNC & Metastasis \\
\hline miR-27b & CYP1B1 & $\begin{array}{l}\text { Modulation of the response of tumor to } \\
\text { anti-cancer drugs }\end{array}$ \\
\hline miR-126 & IRS-1 & Cell cycle progression from G1/G0 to S \\
\hline miR-101 & EZH2 & oncogenic and metastatic activity \\
\hline miR-145 & miR-145 in p53-mediated & $\begin{array}{l}\text { Suppresses Cell Invasion and } \\
\text { repression of c-Myc. }\end{array}$ \\
\hline miR-146a/b b & NF-KB & $\begin{array}{l}\text { negatively regulate factor-KB, and } \\
\text { impaired invasion and migration } \\
\text { capacity }\end{array}$ \\
\hline miR-205 & ErbB3 and VEGF-A expression & $\begin{array}{l}\text { inhibits tumor cell growth and cell } \\
\text { invasion, }\end{array}$ \\
\hline Oncogenic miRNAs & Apoptosis \\
\hline miR-21 & $\begin{array}{l}\text { BCL- } \\
\text { 2,TPM1,PDCD4,PTEN,MASPIN }\end{array}$ & TGF-beta signaling \\
\hline miR-155 & RHOA & Metastasis \\
\hline miR-10b & HOXD10 & $\begin{array}{l}\text { Cell cycle progression G2- M } \\
\text { checkpoint regulation }\end{array}$ \\
\hline $\begin{array}{l}\text { miR- } \\
373 / 520 c\end{array}$ & CD44 & Tamoxifen resistance \\
\hline miR-27a & Zinc finger ZBTB10, Myt-1 \\
\hline miR221/222 & p27Kip1 & \\
\hline & &
\end{tabular}




\section{Computational Method}

In order to finds miRNAs differentially expressed in breast cancer, we have analyzed two breast cancerrelated data contains gene expression values (GSE57297, GSE10797). The fold change values were then filtered based on the two-fold threshold. Then all differentially expressed miRNAs were extracted from the list and considered to be important in cancer. For enriching the results, KEGG database was also scanned to find cancer-related miRNAs. Among the list of all important miRNAs, those related to breast cancer at different stages were extracted and considered to be important. A comparison between these two sets of findings identify the most significant miRNAs involved in different stages of breast cancer development. Table 2 and table 3 shows the results of KEGG and Microarray data analysis for breast cancer. The results indicated that although some miRNAs including miR7 and let7 are shared among these two sets of analysis, many miRNAs are found in only one of dataset. It could be due to variation between samples and the stages that samples were collected.

\section{Table (2) Documentation of miRNAs involved in breast cancer using KEGG}

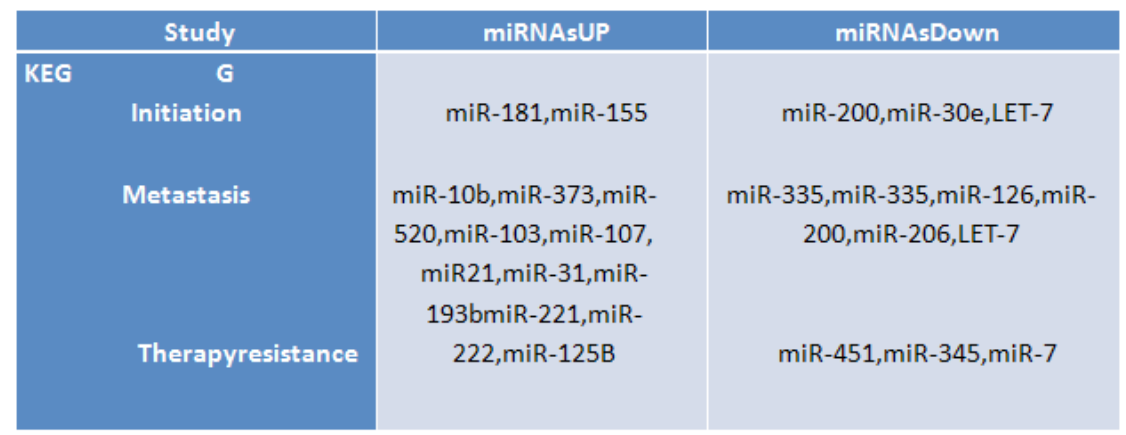

Table (2) Identification of miRNAs involved in breast cancer revealed by gene expression data analysis

\begin{tabular}{|c|c|c|}
\hline Dataset & Up-regulated & Down-regulated \\
\hline NCBI(gse57297) & $\begin{array}{l}\text { miR-7-3p,miR-7-5p,miR- } \\
155, \mathrm{miR}-155\end{array}$ & $\begin{array}{l}\text { miR-100,miR-100-LTE-7a-2 } \\
\text { dusterhostgene,miR-143,miR- } \\
\text { 143hostgene,miR-100,miR- } \\
\text { 143,miR-205,miR-204,miR- } \\
\text { 8071-1, } \\
\text { miR-6743R, }\end{array}$ \\
\hline NCBI(gse10797) & $\begin{array}{l}\text { miR-6132,miR-7703,miR- } \\
\text { 3656,miR-4640,miR- } \\
\text { 4738,miR-3636, } \\
\text { miR6734,miR-3636R,miR- } \\
\text { 3620R,miR- }\end{array}$ & $\begin{array}{l}\text { miR-8071-2R,miR- } \\
\text { 8071,miR6743R,miR- } \\
\text { 6878,miR637R,miR-4746R,miR- } \\
\text { 742,miR3917,miR-600R-miR- } \\
\text { 600, }\end{array}$ \\
\hline
\end{tabular}

\section{Conclusion}

The present research was demonstrated the impact that different method of analysis have on circulating miRNAs. There is a need for standardization in approaches taken to analyses circulating miRNAs. Researchers need to be transparent in reporting of findings to allow for comparison of results and improved reproducibility.

This study has abridged the function of miRNAs in cancer biology, through a particular focus on breast cancer. Sympathetic the molecular mechanisms convoluted in miRNA expression and secretion and expression of miRNA in body fluids and different tissues, were significant subjects in both basic and clinical research. Overall results, highlighted the role of miRNAs during different stages of breast cancer development. The precise role of each miRNA remains to be further investigated. It is also difficult to determine if the differentially expressed miRNAs are cause or effects of breast cancer. 
Ethical Clearance: The Research Ethical Committee at scientific research by ethical approval of both MOH and MOHSER in Iraq

\section{Conflict of Interest: None}

Funding: Self-funding

\section{References}

1. Singh R and Mo Y. Role of microRNAs in breast cancer cancer biology and theory . 2013; 14(3): 201-212

2. Masood u, Rehman $\mathrm{K}$ and etls,"Role of miRNAs in Breast Cancer Asian Pacific J Cancer Prev. 2011; V(12), 3175-3180.

3. Croce $\mathrm{C}$ M. Are circRNAs involved in cancer pathogenesis Cell. 2016; 165, 289-302.

4. Michael G, Schrauder and etls. Circulating MicroRNAs as Potential Blood-Based Markers for Early Stage Breast Cancer Detection . PLoS ONE January. 2012; V (7), Issue 1, e29770

5. Jemal A, Bray F, Ward E and Center M. Global Cancer Statistics. Cancer J. Clin. 2018; 68(6):394424.

6. Iraqi Cancer Board. Results of Iraqi Cancer Registry 20042005. Ministry of Health. BaghdadIraq. 2015.

7. Ng E, Rufina L, Vivian $\mathrm{Y}$, Hong $\mathrm{C}$ and Candy P. Circulating miRNAs as specific biomarker for breast cancer detection. PLoS One. 2013; 8: e53141.

8. BAL H and Hujol J . JAVA for bioinformatics and biomedical applications. Springer-Velage/NY. 2007.

9. Bartels C and Tsongalis G. MicroRNAs: Novel biomarkers for human cancers. Clin Chem. 2009; 55: 623-631.

10. Salsow D, Boetes C, Burke W, Harms S and Russell C. American Cancer Society Breast Cancer advanced group. American Cancer Society guide lines for breast screening with MRI as an adjunct to mammography. CA Cancer J Clin. 2007; 57: 75-89.

11. Harris L, Fritsche H, Mennel R, Norton L, Ravdin $\mathrm{P}$ and Taube S. American Society of Clinical Oncology 2007 update of recommendations for the use of tumor markers in Breast Cancer. J Clin Oncol. 2007; 25: 5287-5312.

12. Lee R and Ambros V. An extensive class of small
Medico-legal Update, April-June 2021, Vol. 21, No. 2

1459

RNAs in C. elegans Science. 2001; 294: 862-864.

13. Ambros V. The functions of animal microRNAs. Nature. 2004; 431: 350-355.

14. Nilsen T. Mechanisms of miRNA- mediated gene regulation in animal cell. Trends. Genet. 2007. 23: 243-249.

15. Ferlay J, Steliarova-Foucher E, Lortet- Tieulent, J, Rosso S, Coebergh J and Comber H . Cancer incidence and mortality patterns in Europe: Estimates for 40 countries in 2012. European J. Ca. 2013; 49: 1374-1403.

16. DeSantis C, Howlader N, and Cronin K . Breast cancer incidence rates in US women are no longer declining. Ca. Epidemiol Biomarkers Prev. 2011; 20: 733-739.

17. Irland National Cancer Registry. Irland National Cancer Registry. National Cancer Registry, Irland Cancer Projections, 20052035. 2008.

18. Calin G, Ferracin M, Cimmino A, Di Leva G, Shimizu M and Wojcik S. A microRNA signature associated with prognosis and progrssion in chronic lympocytic leukemia. N. Engl. J. Med. 2005; 353: 1793-1801.

19. Calin G, Liu C, Sevignani C, Ferracin M, Felli $\mathrm{N}$ and Dumitru C. MicroRNA profiling reveals distinct signitures in B cell chronic lymphocytic leukemias. Proc. Natl. Acad. Sci. USA. 2004; 101: 11755-11760.

20. Bartels $\mathrm{C}$ and Tsongalis G . MicroRNAs: Novel biomarkers for human cancers. Clin Chem. 2009; 55: 623-631. 5

21. Chen $\mathrm{H}$ and Kumar S . Hox genes: emerging stars in cancer. Ca. Biol. Their. 2003; 2: 524-525.

22. Lu J, Getz G, Miska E, Lamb G, and Golub T. MicroRNA expression profiles classify human cancers. Nature. 2005; 435, 834-838.

23. Miska E . How miRNA control cell division, differentiation and death. Curr. Opin. Genet. 2005; 15: 653-658.

24. Lou Y, Yang X, Wang F, Cui Z and Huang Y. MicroRNA 21 promotes the cell proliferation, invasion and migration abilities in ovarian epithelial carcinomas through inhibiting the expression of PTEN protein. Int. J. Mol. Med. 2010; 26: 819-827.

25. Li Y, Wang $Y$, Shen $X$ and Han X. miR-128 Functions as an OncomiR for the Downregulation of HIC1 in Breast Cancer. 2019; v(10), article 1202. 
26. Selcuklu S, Donoghue M, and Spillane C. MiR-21 as a key reulator of oncogenic process. Biochem. Soc. Trans. 2009; 37: 918-925.

27. Si M, Zhu S, Wu H, Lu Z and Mo Y. MiR-21 mediated tumor growth. Oncol. 2007; 26: 27992803.

28. .Wang V and Wei W. miRNA: A new player in breast cancer development. J. Ca. Mol. 2007; 3: 133-138.

29. Qian B. High miR-21 expression in breast cancer associated with poor disease free survival in early stage disease and high TGFbeta 1. Breast Ca. Res. Treat. 2009; 117: 131-140.

30. Wang V and Wei W. miRNA: A new player in breast cancer development. J. Ca. Mol. 2007; 3: 133-138.

31. Huang Q, Gumiraddy K, Schrier M, Lesage C, Nagel R and Nair S. The miRNA-373 and miR$520 \mathrm{c}$ promote tumor invasion and metastasis. Nat. Cell Biol. 2008; 10: 202-210.
32. Zhao H, Shen J, Medico L, Wang D, and Ambrosome C. A pilot study of circulating miRNAs as potential biomarkers of early stage breast cancer. PLoS One. 2010; 5: e13735.

33. Schrauder M, Strick R, Schulz-Wendtland R, Strissel P, Kahmann L and Loehberg C. Circulating microRNAs as potential blood based markers for early stage breast cancer detection. PLoS ONE. 2012; 7: e29770.

34. Heneghan H, Miller N, Kelly R, and Newell J. Systemic miR-195 diffrentiate breast cancer from other malignancies and is a potential biomarker for detecting noninvasive and early stage disease. Oncologist. 2010; 15: 673-682.

35. Si H, Sun X, Chen Y, Cao Y, Chen S and Wang H. Circulating microRNA-92a and microRNA-21 as novel minimally invasive biomarkers for primary breast cancer. J. Ca. Res. Clin. Oncol. 2013; 139: 223-229. 